\title{
Salamander Hox clusters contain repetitive DNA and expanded non-coding regions: a typical Hox structure for non-mammalian tetrapod vertebrates?
}

\author{
Stephen Randal Voss ${ }^{1,2^{*}}$, Srikrishna Putta ${ }^{1,2}$, John A Walker ${ }^{1,2}$, Jeramiah J Smith', Nobuyasu Maki ${ }^{3}$ \\ and Panagiotis A Tsonis ${ }^{4}$
}

\begin{abstract}
Hox genes encode transcription factors that regulate embryonic and post-embryonic developmental processes. The expression of Hox genes is regulated in part by the tight, spatial arrangement of conserved coding and non-coding sequences. The potential for evolutionary changes in Hox cluster structure is thought to be low among vertebrates; however, recent studies of a few non-mammalian taxa suggest greater variation than originally thought. Using next generation sequencing of large genomic fragments (>100 kb) from the red spotted newt (Notophthalamus viridescens), we found that the arrangement of Hox cluster genes was conserved relative to orthologous regions from other vertebrates, but the length of introns and intergenic regions varied. In particular, the distance between hoxd13 and hoxd11 is longer in newt than orthologous regions from vertebrate species with expanded Hox clusters and is predicted to exceed the length of the entire HoxD clusters (hoxd13-hoxd4) of humans, mice, and frogs. Many repetitive DNA sequences were identified for newt Hox clusters, including an enrichment of DNA transposon-like sequences relative to non-coding genomic fragments. Our results suggest that Hox cluster expansion and transposon accumulation are common features of non-mammalian tetrapod vertebrates.
\end{abstract}

Keywords: Hox, Salamander, Genome, Evolution

\section{Background}

Bilaterian body plans are determined in part by DNA transcription factors called Hox genes [1-4]. Excepting fish, vertebrate Hox genes are ordered among four unlinked clusters that each span relatively short segments of genomic DNA (generally 100-200 Kb). The arrangement of Hox genes on chromosomes is co-linear with their pattern of transcription along the anteriorposterior and proximal-distal body axes during embryonic development $[5,6]$. The organization and structure of $H o x$ gene clusters and associated non-coding regulatory elements are mostly conserved across vertebrates $[7,8]$. However, as genomic studies extend to non-

\footnotetext{
*Correspondence: srvoss@uky.edu

'Department of Biology, University of Kentucky, Lexington, KY 40506, USA

${ }^{2}$ Spinal Cord and Brain Injury Research Center, University of Kentucky,

Lexington, KY 40506, USA

Full list of author information is available at the end of the article
}

genetic model organisms, variations in Hox cluster structure are being discovered, including variations in gene number, repetitive sequence content, cluster length, and non-coding sequence conservation [9-15]. These variations suggest that the evolution of Hox cluster structure may correlate with phylogeny, unique modes of vertebrate development, and/or derived morphological characteristics.

In tetrapod vertebrates, stereotypic patterns of Hox expression are observed along the proximal-distal axes of developing limbs [16]. In most species, Hox developmental genetic programs are only expressed during limb development. However, salamanders reactivate Hox gene expression throughout life to correctly pattern tissues within regenerating limbs [17-21]. While some patterns of Hox expression in regenerating limbs recapitulate the expression pattern in developing limbs, spatial and temporal differences are observed [18-21]. This raises the 
possibility that salamander Hox clusters may contain non-coding elements that uniquely regulate postembryonic, tissue regeneration; such elements may not be expected within Hox clusters of vertebrates incapable of limb regeneration. There is another reason to suspect that salamander Hox clusters may differ from other vertebrate taxa-salamanders as a group have extremely large genomes. An average sized salamander genome is approximately $10 \times$ larger than the Homo sapiens genome; some salamanders have genomes that are 30× larger [22]. This larger genome size is reflected in the structure of genes, as salamander introns are longer on average than orthologous introns in other vertebrates [23,24].

Belleville et al. [25] reported that two pairs of adjacent Hox cluster genes from the red spotted newt (Notophthalamus viridescens) presented highly conserved coding and noncoding sequences relative to orthologous mammalian Hox sequences. These results suggested that Hox cluster evolution is constrained even within the context of a very large vertebrate genome (>20 pg/haploid nucleus) [22]. However, the results that we present below show that newt Hox clusters are more variable than originally thought. Sequencing of large genomic fragments $(>100 \mathrm{~Kb})$ reveals regional variation in length across newt Hox cluster regions and higher proportions of DNA transposon-like sequences within Hox introns and intergenic sequences than non-coding genomic regions. Our results show that expanded non-coding regions and relatively high repetitive DNA sequence content are typical of Hox clusters in amphibians and other non-mammalian tetrapod vertebrates.

\section{Results and discussion}

\section{BAC library screening, sequencing, assembly, and} annotation

A bacterial artificial clone (BAC) library of 41,472 clones was constructed for newt, and pools were screened by polymerase chain reaction (PCR) to identify clones that contained Hox genes. Two BACs containing HoxC (NV_H3_75P19; [GenBank:JF490017.1]) and HoxD (NV_H3_85F1; [GenBank: JF490018.1]) orthologs and two additional BACs containing only non-coding genomic DNA (NV_H3_28J3; [GenBank: JF490019.1] and NV_H3_32L5; [GenBank:JF490020.1]) were purified and sequenced to an average depth of 220 bp sequence reads per nucleotide position. The reads for NV_H3_75P19 were assembled into three large contigs with the breaks occurring between hoxc5 and hoxc4, and a position $3^{\prime}$ of hoxc4. The reads for NV_H3_28J3 were reassembled into two large contigs with the break occurring $5^{\prime}$ of hoxd11. The reads for the BACs that contained noncoding genomic DNA generated more than three contigs and could not be ordered; these were randomly concatenated for analyses described below. BLASTx searches revealed that the BACs containing Hox sequences contained some, but not all of Hox gene members from each cluster:
NV_H3_75P19 contained hoxc11, hoxc10, hoxc9, hoxc8, hoxc6, hoxc5, and hoxc4, and NV_H3_85F1 contained hoxd11, hoxd10, hoxd9, and hoxd8. The order of newt Hox genes was conserved relative to orthologs in other vertebrate genomes, as were coding and non-coding sequences, and exon/intron boundaries (Figures 1 and 2). High sequence identity was observed for Hox genes, which is typical of transcription factors that function in highly conserved developmental pathways. Conserved non-coding sequences (CNS) were identified from regions flanking Hox exons; these likely correspond to enhancer elements and non-coding RNAs that function in the regulation of Hox gene expression. For example, two CNSs that were identified downstream of newt hoxd11 (40 kb) correspond to enhancer elements VIII and IX from Gerard et al. [26], and a CNS upstream (3 kb) of newt hoxc8 corresponds to an enhancer from Shashikant et al. [27]. Also, a CNS identified downstream of hoxc10 (28 $\mathrm{kb}$ ) corresponds to human miRNA-196a, and a canonical miR-196a seed-pairing site is predicted $268 \mathrm{bp}$ from the end of newt hoxd8 [28]. Thus, elements that are known to regulate $H o x$ gene functions in other vertebrate species show identity to sequences in newt Hox genomic regions.

While the general organization of newt Hox genes was conserved relative to other vertebrates, extensive variation was observed in the lengths of intergenic and intronic sequences (Table 1; Figures 3 and Figure 4). The length of the newt hoxc11-c4 region was longer than orthologous mammalian (H. sapiens, Mus musculous) and zebrafish (Danio rerio) regions, but shorter than regions from Anolis carolinensis (lizard) and Xenopus tropicalis (frog), which are known to have expanded $H o x C$ clusters [13]. While newt HoxC introns were also longer than mammalian and fish introns, frog and lizard introns also exceed the length of their mammalian counterparts. This supports the idea that salamander genes typically contain long introns $[23,24]$, although we did not observe the same pattern for $H o x D$ genes. While long lizard and frog HoxD introns were observed, newt hoxd11-9 introns were typically shorter than orthologous mammalian introns. Thus, while non-mammalian tetrapod vertebrates, and especially the anolis lizard, have Hox genes with long introns, relative intron length varies among paralogous members of newt HoxD and HoxC clusters.

In annotating HoxD cluster genes, we discovered that hoxd11 was located approximately $73 \mathrm{~kb}$ from the terminus of NV_H385F1. This distance, which provides a minimum estimate to the expected position of hoxd13 (hoxd12 is not known for amphibians [15,26]), predicts the newt hoxd13-11 segment to be $>4.5 \times$ and $1.5 \times$ longer than orthologous $H o x D$ regions from frog and lizard. It also exceeds the length of hoxd11-13 segments in the coelacanth and a caecilian amphibian (Typhlonectes natans) [29], which until this study was thought to be longest among vertebrates (Figure 4). While it is possible 


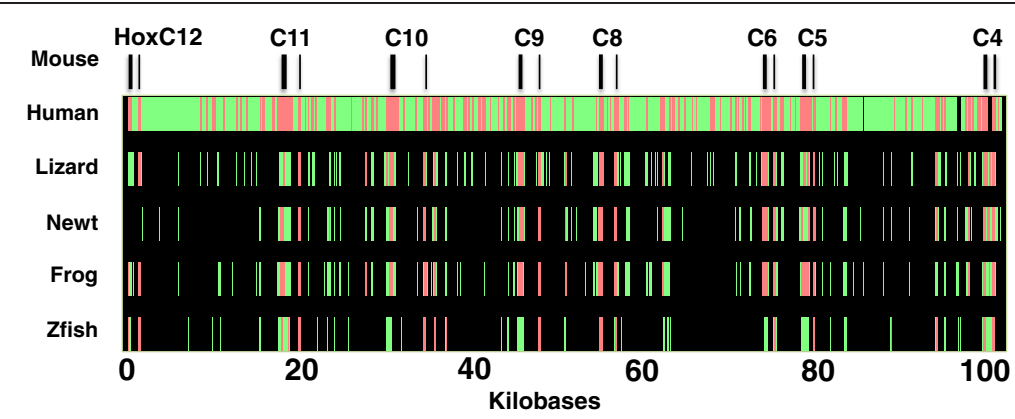

Figure 1 Multispecies alignment of orthologous HoxC genomic regions among selected vertebrate species. The black bars at the top of the figure show the positions of exons in the mouse sequence, which was used as the reference sequence for alignment. Red bars indicate strongly aligned regions - at least 100 bp in length without a gap and $>70 \%$ nucleotide identity. Green bars indicate all other aligned regions. Zfish, zebrafish.

that the expanded region is explained by an evolutionary loss of the newt hoxd13 gene, this seems unlikely because hoxd13 orthologs are known for related salamanders [15], and we did not detect the presence of a pseudogene nucleotide signature. Because expanded Hox clusters have been shown for a representative caecilian [26] and anuran species [13], parsimony suggests the expansion of the hoxd11-13 region to be a shared derived characteristic of amphibians, with convergent expansion of the same region in lizard.

\section{Interspersed repeat sequences in BACs}

Previous studies have shown that interspersed repetitive DNA sequences are rarely observed within Hox clusters of mammals and some reptiles, but are more abundant in species with expanded Hox clusters [15,26]. To test this idea, we searched Hox and non-Hox genomic clones for repeats that are catalogued in RepBase (Genetic Information Research Institute, Mountain View, USA) [30], and also aligned genomic sequences using MultiPipmaker [31] to identify direct and indirect repeats unique to the newt. In many cases, we found that both approaches identified repetitive sequences for the same segments of DNA; however, more newt specific repeats were identified overall (Additional file 1: Table S1). The annotated (i.e., RepBase) interspersed repetitive sequence content of HoxC and HoxD genomic sequences was approximately two to three times lower than the content of the two, non-protein coding genomic clones (Table 2). Considering annotated and newt-specific repeats, $77 \%$ of the noncoding genomic sequence was identified as repetitive, compared to $24 \%$ and $32 \%$ for HoxC and HoxD sequences (Additional file 1: Table S1). These results suggest that the fixation probability for repetitive element accumulation is lower for Hox clusters, presumably because these regions are evolutionarily constrained by the functional sequences they encode. Repeats were more frequent in regions flanking genes, with the large intergenic regions flanking terminal Hox loci showing the greatest accumulation (Figures 3 and 4). Repeats were predicted for introns, and a higher density of DNA transposon-like sequences were predicted within $H o x C$ and $H o x D$ clusters than within non-coding genomic clones. Interestingly, the enrichment of DNA transposon-like sequences was about 20-fold for $H o x C$ but only 2-fold for HoxD (Table 2). While this may reflect sampling bias, the more expanded of the two newt

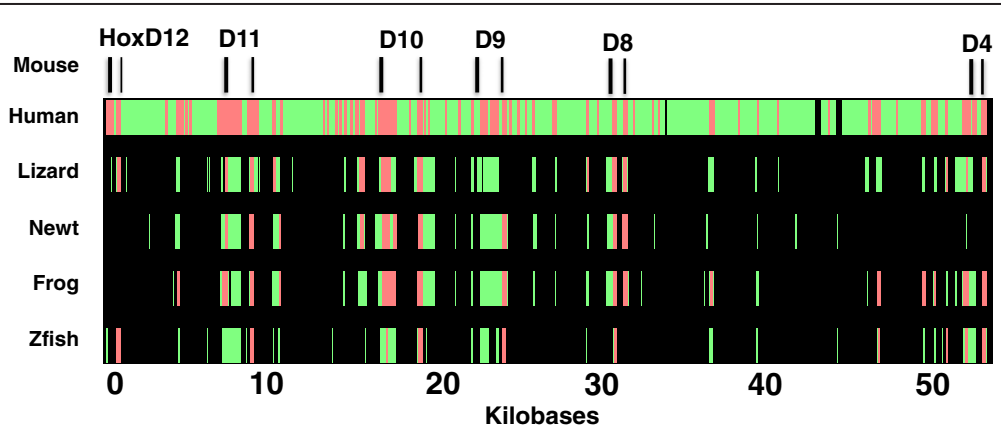

Figure 2 Multispecies alignment of orthologous HoxD genomic regions among selected vertebrate species. The black bars at the top of the figure show the positions of exons in the mouse sequence, which was used as the reference sequence for alignment. Red bars indicate strongly aligned regions - at least 100 bp in length without a gap and >70\% nucleotide identity. Green bars indicate all other aligned regions. Zfish, zebrafish. 
Table 1 Species comparison of HoxC and HoxD intron lengths

\begin{tabular}{lllllll}
\hline Gene ID & Human & Mouse & Lizard & Frog & Newt & Zebrafish \\
\hline hoxc11 & 1257 & 1261 & 2025 & 1334 & 2907 & 1368 \\
hoxc10 & 3158 & 3189 & 4591 & 2498 & 3211 & 1766 \\
hoxc9 & 1703 & 1704 & 2139 & 1560 & 2225 & 1059 \\
hoxc8 & 1368 & 1347 & 2075 & 1619 & 1393 & 1265 \\
hoxc6 & 733 & 728 & 902 & 763 & 1283 & 610 \\
hoxc5 & 701 & 692 & 927 & 713 & 753 & 818 \\
hoxc4 & 488 & 475 & 1006 & 448 & 502 & 520 \\
hoxd11 & 770 & 735 & 1667 & 748 & 675 & 863 \\
hoxd10 & 1375 & 1366 & 1628 & 1980 & 1233 & 715 \\
hoxd9 & 348 & 346 & - & 341 & 345 & 316 \\
hoxd8 & 373 & 395 & 2474 & 1733 & 434 & - \\
\hline
\end{tabular}

The longest intron is italicized for each Hox gene.

Hox clusters does not contain a higher proportion of DNA transposon-like sequences; instead, HoxD contains a moderately higher proportion of long interspersed retroelement-like sequences, simple repeats, and newtspecific repeats. Observation of a higher frequency of DNA transposon-like sequences, within arguably a more functionally constrained $\mathrm{HoxC}$ cluster, suggests an insertion bias for Hox genic regions. While this speculation awaits further study, our results support the idea that repetitive sequences, and in particular DNA transposon-like sequences, are more abundant within Hox clusters of nonmammalian tetrapod vertebrates [13] than is indicated by analysis of mammalian Hox clusters.

\section{Conclusions}

Salamander Hox genomic regions show elements of conservation and diversity in comparison to other vertebrate species. Whereas the structure and organization of Hox coding genes is conserved, newt Hox clusters show variation in the lengths of introns and intergenic regions, and the hoxd13-11 region exceeds the lengths of orthologous segments even among vertebrate species with expanded Hox clusters. We posit that the hoxd1311 expansion predated a basal salamander genome size increase that occurred approximately 180 million years ago [32] as it is preserved in all three extant amphibian groups. Over more recent timescales, additional evidence supports the idea that Hox clusters are amenable to structural evolution: there is variation in the lengths of introns and intergenic regions, relatively high numbers of repetitive sequences, and non-random accumulations of DNA transposons in newts and lizards. The non-random accumulation of DNA-like transposons could potentially alter developmental programming by creating sequence motifs for transcriptional regulation [33-35]. Overall, available data from several nonmammalian tetrapods suggest that Hox structural flexibility is the rule, not the exception. We speculate that such flexibility may contribute to developmental variation across non-mammalian taxa, both in embryogenesis and during the re-deployment of Hox genes during post-embryonic developmental processes, such as metamorphosis and regeneration.

\section{Methods}

\section{BAC library construction, screening, and sequencing}

The Clemson University Genomics Institute constructed a BAC library from partially restriction digested and size-selected genomic DNA that was isolated from the erythrocytes of a single Notophthalamus viridescens female (University of Dayton Institutional Animal Care and Use Committee Protocol \# 011-12). A total of 41,472 clones were arrayed in $108 \times 384$ well plates. Superpools of clones were made by combining clones from twelve 384 well plates into a single pool. DNA was extracted from $400 \mathrm{ml}$ of overnight cultures of

(A)

(B)

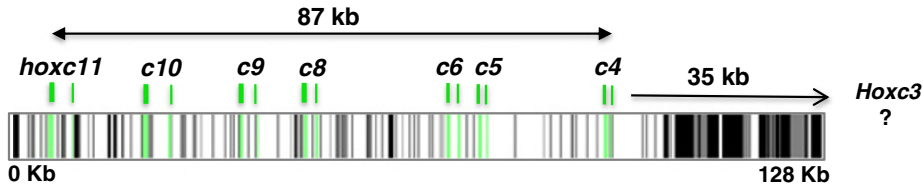

)

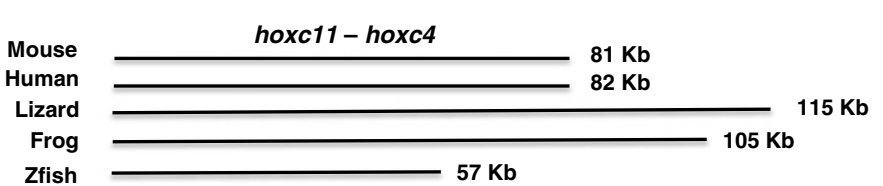

Figure 3 Structure and repetitive sequence content of newt HoxC, and total length of orthologous hoxc11-c4. (A) The structure and repetitive sequence content of newt HoxC. Green bars indicate the positions of exons, gray bars indicate the positions of RepBase repeats, and black bars indicate the positions of unique newt repetitive sequences. (B) The total length of orthologous hoxc11-c4 non-coding segments from representative vertebrates. 


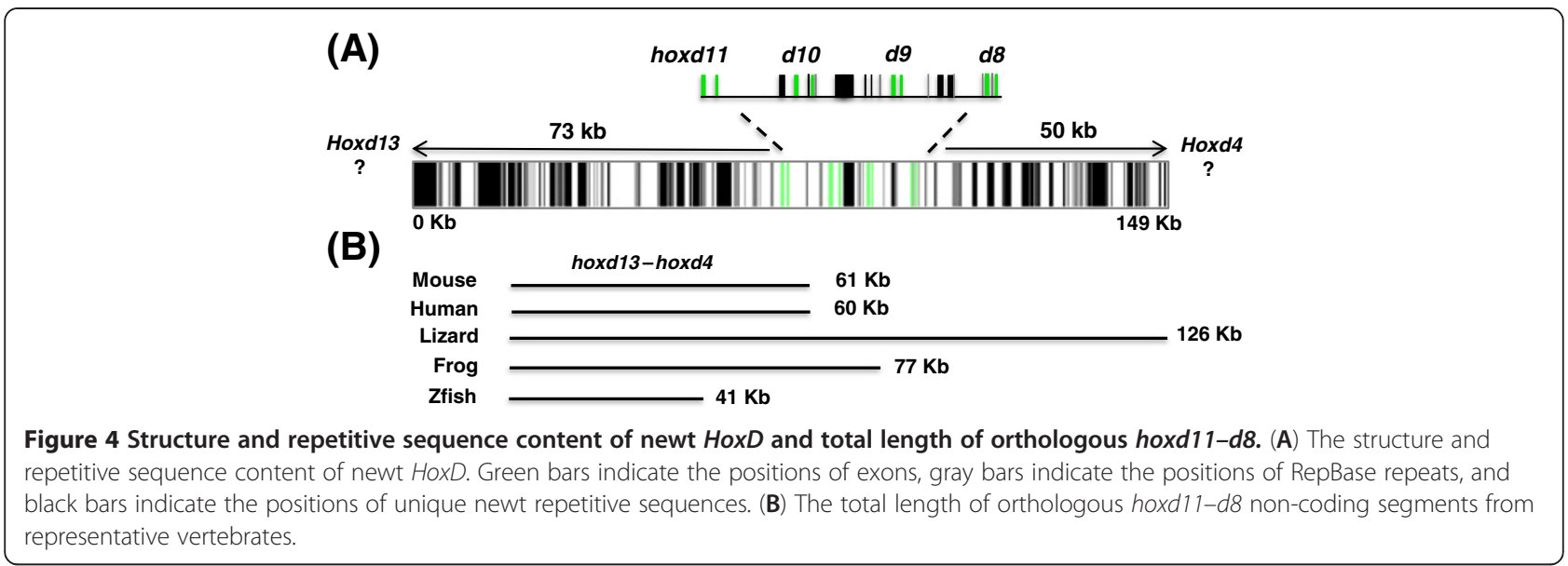

superpools using the Plasmid MaxiPrep kit (Qiagen, Valencia, CA, USA), and the DNA pellet was resuspended in $250 \mu \mathrm{l}$ of water. PCR primers for newt hoxc10 (forward: CAAAGAGAAAACGCGGAAAG; reverse: CGATACCGTCCCTTCCATAA) and hoxd10 (forward: TTTCCATTGTCGGTTTTTCC; reverse: TCCT ACCACGGACATTACCC) were used to identify two Hox gene-containing BACs and two BACs that did not contain protein-coding sequence. The four clones were grown in $400 \mathrm{ml} \mathrm{L}$-broth, and DNA was isolated using the Qiagen Large Construction Kit (Qiagen); genomic DNA contamination was reduced using Plasmid-Safe DNAse treatment (Epicentre Biotechnologies, Madison, USA). The Roche GS FLX Titanium platform (Basel, Switzerland) was used to sequence BACs; the work was accomplished by the staff of the University of Iowa Sequencing Core Facility. The termini of BAC inserts were endsequenced using Sanger technology and ABI Big-Dye 3.1 (Invitrogen, Grand Island, USA).

Table 2 Percent coverage of salamander genomic sequences by newt-specific and RepBase repetitive elements

\begin{tabular}{llll}
\hline & HoxC & HoxD & Non-coding \\
\hline Newt-specific repeats & 24 & 32 & 77 \\
Total RepBase repeats & 5.34 & 4.25 & 13.34 \\
Long terminal repeat retrotransposons & 2.75 & 2.70 & 9.50 \\
Non-long terminal repeat retrotransposons & 2.30 & 3.92 & 2.42 \\
DNA transposons & 2.54 & 0.32 & 0.12 \\
Unclassified & 0.04 & 0.00 & 1.30 \\
Satellites & 0.00 & 0.50 & 0.31 \\
Simple repeats & 0.88 & 1.88 & 0.87 \\
Low complexity & 0.62 & 0.38 & 0.31 \\
\hline
\end{tabular}

DNA sequence assembly and annotation

Sequences were screened to trim vector, adapters, and contaminating Escherichia coli sequences. After an initial assembly using GS De Novo Assembler (454 Life Sciences, Branford, USA), contigs and singletons were assembled further using DNASTAR SeqMan (DNASTAR, Inc., Madison, USA). Contiguous sequences of assembled BACs were searched (blastn) against salamander expressed sequence tagged contigs at Sal-Site [36]; non-redundant nucleotide and protein databases at NCBI (blastx and tblastp) [37] were used to identify and annotate gene regions. For multispecies comparisons, genomic sequences for H. sapiens (GRCh37.10), and M. musculus (GRCh38.1), were obtained from NCBI. Anolis carolinesis (AnoCar 2.0) and D. rerio $(\mathrm{Zv} 9)$ were obtained from Ensembl [38]. X. tropicalis (build 7.1) was obtained from Xenbase [39]. Sequences were aligned using MultiPipMaker [28]. Annotated repeats were identified by searching re-assembled BAC clones against all deposited repeats in RepBase [30]. Newt-specific repeats were identified using MultiPipmaker [28] by aligning re-assembled BAC clones against each other and by performing self-self BAC alignments. The "search both strands" and "high sensitivity" options were used in MultiPipmaker to identify significantly similar noncoding sequences that are located to different positions either within or between BACs. The terminal base pair positions for these alignments were recorded to denote the positions of repetitive sequences within BACs. If the two repeats occurred within $50 \mathrm{bp}$ of each other, they were compiled as a single repetitive sequence with the most terminal base positions denoting the repeat span. The base pair coordinates for newt-specific repetitive sequences were combined with base pair coordinates for RepBase repetitive sequences to generate an underlay file (Additional file 1: Table S1), and this was used to create maps of repetitive elements for the $\operatorname{HoxD}$ and $\operatorname{HoxC}$ genomic regions. 


\section{Additional file}

Additional file 1: Table S1. Base pair coordinates for RepBase and newt-specific repeats identified from BAC clones with HoxC and HoxD genomic regions.

\section{Competing interest}

The authors declare that they have no competing interests.

\section{Authors' contributions}

SRV, JJS, NM, and PAT conceived and designed the experiment, and analyzed and interpreted the results. SP assembled sequence reads corresponding to BACs and analyzed and annotated the sequence data. JAW performed PCR, isolated BACs for sequencing, and analyzed and annotated the sequence data. SRV drafted the manuscript. All authors read and approved the final manuscript.

\section{Acknowledgments}

The research was supported by grant R24-OD010435 (SRV) and EY-10540 (PAT) from the National Center for Research Resources (NCRR), a component of the National Institutes of Health $(\mathrm{NIH})$. The project also used resources developed under Multidisciplinary University Research Initiative grant (W911NF-09-1-0305) from the Army Research Office (SRV) and resources from the Ambystoma Genetic Stock Center, which is funded by grant DBI-0951484 from the National Science Foundation (SRV). The contents of this paper are solely the responsibility of the authors and do not necessarily represent the official views of NCRR, NIH, ARO, or NSF.

\section{Author details}

${ }^{1}$ Department of Biology, University of Kentucky, Lexington, KY 40506, USA ${ }^{2}$ Spinal Cord and Brain Injury Research Center, University of Kentucky, Lexington, KY 40506, USA. ${ }^{3}$ Institute of Protein Research, Osaka University, 3-2 Yamadaoka, Suita-Shi, Osaka 565-0871, Japan. ${ }^{4}$ Department of Biology, University of Dayton, Dayton, $\mathrm{OH}$, USA.

Received: 10 January 2013 Accepted: 25 January 2013 Published: 5 April 2013

\section{References}

1. Lewis EB: A gene complex controlling segmentation in Drosophila. Nature 1978, 276:565-570.

2. Krumlauf R: Hox genes in vertebrate development. Cell 1994, 78:191-201.

3. Duboule D: The rise and fall of Hox gene clusters. Development 2007 134:2549-2560.

4. Lemons D, McGinnis W: Genomic evolution of Hox gene clusters. Science 2006, 313:1918-1922.

5. Kmita M, Duboule D: Organizing axes in time and space; 25 years of colinear tinkering. Science 2003, 301:331-333.

6. Tschopp P, Duboule D: A genetic approach to the transcriptional regulation of Hox gene clusters. Annu Rev Genet 2011, 45:145-166.

7. Garcia-Fernandez J: The genesis and evolution of homeobox gene clusters. Nat Rev Genet 2005, 6:881-892.

8. Duboule D: Temporal colinearity and the phylotypic progression: a basis for the stability of a vertebrate Bauplan and the evolution of morphologies through heterochrony. Dev Suppl 1994, 1994:135-142.

9. Aparicio S, Chapman J, Stupka E, Putnam N, Chia JM, Dehal P, Christoffels A, Rash S, Hoon S, Smit A, Gelpke MD, Roach J, Oh T, Ho IY, Wong M, Detter C, Verhoef F, Predki P, Tay A, Lucas S, Richardson P, Smith SF, Clark MS, Edwards YJ, Doggett N, Zharkikh A, Tavtigian SV, Pruss D, Barnstead M Evans $C$, et al: Whole-genome shotgun assembly and analysis of the genome of Fugu rubripes. Science 2002, 297:1301-1310.

10. Hoegg S, Meyer A: Hox clusters as models for vertebrate genome evolution. Trends Genet 2005, 21:421-424.

11. Kurosawa G, Takamatsu N, Takahashi M, Sumitomo M, Sanaka E, Yamada K, Nishii K, Matsuda M, Asakawa S, Ishiguro H, Miura K, Kurosawa Y, Shimizu N, Kohara $Y$, Hori H: Organization and structure of Hox gene loci in Medaka genome and comparison with those of pufferfish and zebrafish genomes. Gene 2006, 370:75-82.

12. Woltering JM, Vonk FJ, Müller H, Bardine N, Tuduce IL, de Bakker MAG, Knöchel W, Sirbu IO, Durston AJ, Richardson MK: Axial patterning in snakes and caecilians: evidence for an alternative interpretation of the Hox code. Dev Biol 2009, 332:82-89.

13. Di-Poi N, Montoya-Burgos J, Duboule D: A typical relaxation of structural constraints in Hox gene clusters of the green anole lizard. Genome Res 2009, 19:602-610.

14. Matsunami M, Sumiyama K, Saitou N: Evolution of conserved non-coding sequences within the vertebrate Hox clusters through the two-round whole genome duplications revealed by phylogenetic footprinting analysis. J Mol Evol 2010, 71:427-436.

15. Liang D, Wu R, Geng J, Chaolin Wang C, Zhang P: A general scenario of Hox gene inventory variation among major sarcopterygian lineages. BMC Evol Biol 2011, 11:25

16. Zakany J, Duboule D: The role of Hox genes during vertebrate limb development. Curr Opin Genet Dev 2007, 17:359-366.

17. Savard P, Gates PB, Brockes JP: Position dependent expression of a homeobox gene transcript in relation to amphibian limb regeneration. EMBO J 1988, 7:4275-4282.

18. Gardiner DM, Blumberg B, Komine Y, Bryant SV: Regulation of HoxA expression in developing and regenerating axolotl limbs. Development 1995, 121:1731-1741

19. Torok MA, Gardiner DM, Shubin NH, Bryant SV: Expression of HoxD genes in developing and regenerating axolotl limbs. Dev Biol 1998, 200:225-233.

20. Khan PA, Tsilfidis C, Liversage RA: Hox C6 expression during development and regeneration of forelimbs in larval Notophthalmus viridescens. Dev Genes Evol 1999, 209:323-329.

21. Carlson MR, Komine Y, Bryant SV, Gardiner DM: Expression of Hoxb13 and Hoxc10 in developing and regenerating axolotl limbs and tails. Dev Biol 2001, 229:396-406.

22. Gregory TR: Animal Genome Size Database 2010. http://www.genomesize. com.

23. Casimir CM, Gates PB, Ross-Macdonald PB, Jackson JF, Patient RK, Brockes JP: Structure and expression of a newt cardio-skeletal myosin gene: implications for the C value paradox. J Mol Biol 1992, 202:287-296.

24. Smith JJ, Putta S, Zhu W, Pao GM, Verma IM, Hunter T, Bryant SV, Gardiner DM, Harkins TT, Voss SR: Genic regions of a large salamander genome contain long introns and novel genes. BMC Genomics 2009, 10:19.

25. Belleville S, Beauchemin M, Tremblay M, Noiseux N, Savard P: Homeoboxcontaining genes in the newt are organized in clusters similar to other vertebrates. Gene 1992, 114:179-186.

26. Gerard M, Duboule D, Zakany J: Structure and activity of regulatory elements involved in the activation of the Hoxd-11 gene during late gastrulation. EMBO J 1993, 12:3539-3550.

27. Shashikant CS, Bolanowsky SA, Anand S, Anderson SM: Comparison of diverged Hoxc8 early enhancer activities reveals modification of regulatory interactions at conserved cis-acting elements. J Exp Zool B Mol Dev Evol 2007, 308:242-249.

28. Hornstein E, Mansfield JH, Yekta S, Hu JK, Harfe BD, McManus MT, Baskerville S, Bartel DP, Tabin CJ: The microRNA miR-196 acts upstream of Hoxb8 and Shh in limb development. Nature 2005, 438:671-674.

29. Mannaert A, Amemiya CT, Bossuyt F: Comparative analyses of vertebrate posterior HoxD clusters reveal atypical cluster architecture in the caecilian Typhlonectes natans. BMC Genomics 2010, 11:658.

30. Smit AFA, Hubley R, Green P: RepeatMasker Open-3.0 1996-2010. http:// www.repeatmasker.org

31. Schwartz S, Zhang Z, Frazer KA, Smit A, Riemer C, Bouck J, Gibbs R, Hardison $\mathrm{R}$, Miller W: PipMaker-a resource for aligning two genomic sequences. Gen Res 2000, 10:577-586.

32. Zhang P, Wake DB: Higher-level salamander relationships and divergence dates inferred from complete mitochondrial genomes. Mol Phylogenet Evol 2009, 53:492-508.

33. Lowe CB, Bejerano G, Haussler D: Thousands of human mobile element fragments undergo strong purifying selection near developmental genes. Proc Natl Acad Sci USA 2007, 104:8005-8010.

34. Mikkelsen TS, Wakefield MJ, Aken B, Amemiya CT, Chang JL, Duke S, Garber M, Gentles AJ, Goodstadt L, Heger A, Jurka J, Kamal M, Mauceli E, Searle SM, Sharpe T, Baker ML, Batzer MA, Benos PV, Belov K, Clamp M, Cook A, Cuff J, Das R, Davidow L, Deakin JE, Fazzari MJ, Glass JL, Grabherr M, Greally JM, Gu W, et al: Genome of the marsupial Monodelphis domestica reveals innovation in non-coding sequences. Nature 2007, 447:167-177.

35. Borque $\mathrm{G}$ : Transposable elements in gene regulation and in the evolution of vertebrate genomes. Curr Opin Genet Dev 2009, 19:607-612. 
36. Smith JJ, Putta S, Walker JA, Kump DK, Samuels AK, Monaghan JR, Weisrock DW, Staben C, Voss SR: Sal-Site: integrating new and existing ambystomatid salamander research and informational resources. BMC Genomics 2005, 6:181.

37. National Center for Biotechnology Information. http://www.ncbi.nlm.nih.gov.

38. Ensembl. http://www.ensembl.org.

39. Xenbase: A Xenopus Laevis and Xenopus Tropicalis Resource. http://www. xenbase.org.

doi:10.1186/1479-7364-7-9

Cite this article as: Voss et al:: Salamander Hox clusters contain repetitive DNA and expanded non-coding regions: a typical Hox structure for non-mammalian tetrapod vertebrates?. Human Genomics 2013 7:9.

\section{Submit your next manuscript to BioMed Central and take full advantage of:}

- Convenient online submission

- Thorough peer review

- No space constraints or color figure charges

- Immediate publication on acceptance

- Inclusion in PubMed, CAS, Scopus and Google Scholar

- Research which is freely available for redistribution 